\title{
Forwarding Strategies for Gaussian Parallel-Relay Networks
}

\author{
Ivana Maric Member, IEEE and Roy D. Yates Member, IEEE
}

\begin{abstract}
This paper investigates reliable and unreliable forwarding strategies in a parallel-relay network. We consider the problem that maximizes the achievable rate under the total power constraint that allows for the power allocation among the nodes. We approach this problem by solving its dual with the objective to communicate to the destination at rate $r$ using minimum transmitted power. Motivated by applications in sensor networks, we assume large bandwidth resources allowing orthogonal transmissions at the nodes. In such a network, the energy cost per information bit [1] during the reliable forwarding is minimized by operating in the wideband regime. For the wideband decodeand-forward (DF) strategy, we present the optimum parallel-relay solution by identifying the best choice of relay nodes and the optimum power allocation among them. We demonstrate that the data should be sent over a single relay route through one relay that is in the "best" position in the network.

On the other hand, as observed in [2], the benefit of unreliable amplify-and-forward (AF) strategy diminishes in the wideband regime. We characterize the optimum bandwidth for AF that minimizes the total energy cost per information bit for our network model. We show that transmitting in the optimum bandwidth allows the network to operate in the linear regime where the achieved rate increases linearly with transmit power. We then identify the best subset of AF relay nodes and characterize the optimum power allocation per dimension among relays, for a given source power and bandwidth.

Based on this analysis, we compare the energy-efficiency of DF and AF in a one-relay network and show the regions where each strategy is optimal.
\end{abstract}

Index Terms - Decode-and-forward, amplify-and-forward, optimum relay strategy, optimum bandwidth

\section{INTRODUCTION}

We consider a Gaussian parallel-relay channel consisting of a single source-destination pair and $M$ relays that dedicate their resources to relaying information for the source. The capacity of this network is not known for any finite $M$. For $M=1$, the capacity was found in [3] for the degraded version of this channel. The asymptotic capacity in the limit as the number of relays $M$ tends to infinity was found in [4], [5], by employing unreliable forwarding at the relays. Another strategy in which relays do not decode a message, but send the compressed received values to the destination, was considered in [3] and extended to a multiple relay channel in [6]. When the relays are close to the destination, this strategy achieves the antenna-clustering capacity [6].

This work was supported by New Jersey Commission on Science and Technology and NSF grant NSF ANI 0338805.

The authors are with Wireless Network Information Laboratory (WINLAB), Department of Electrical and Computer Engineering, Rutgers University, Piscataway, NJ 08854 USA (e-mail: ivanam@winlab.rutgers.edu; ryates@winlab.rutgers.edu).
This approach is in contrast to the strategy where the message is reliably decoded at the relays, enabling relays to re-encode and forward the message to the destination. This strategy achieves the capacity of the degraded relay channel [3]. Its extension to multiple relays was presented in [7], [8]. When relays are close to the source, DF strategy can achieve the capacity in a wireless relay network [8].

This paper investigates reliable and unreliable forwarding strategies in a parallel-relay network. Motivated by applications in sensor networks, we assume large bandwidth resources allowing orthogonal transmissions at the nodes. While the minimum energy cost per information bit in a general relay network is unknown [9], in our model, a decode-and-forward network will minimize the energy cost per information bit [1] by operating in the wideband regime. However, as already observed in [2], the AF strategy should operate in a different regime. A disadvantage of the AF strategy is that the relay power is wasted amplifying the receiver noise at a relay. This effect worsens as the signaling bandwidth increases. In fact, for a network operating in the wideband regime, there is no benefit from relays employing the AF strategy.

With this observation in mind, we investigate the performance of the reliable and unreliable forwarding in a parallelrelay network. We take approach of [10] and consider two dual problems; one that maximizes the achievable rate given the power constraints and the second that minimizes the total average power to support a given rate at the destination node. We present the optimum solution for the wideband DF strategy by identifying the best choice of relay nodes and the optimum power allocation among them. We demonstrate that the data should be sent over a single relay route through one relay that is in the "best" position in the network. We then characterize the optimum bandwidth for AF and show that transmitting in the optimum bandwidth allows the network to operate in the linear regime where the achieved rate increases linearly with transmit power. We then identify the best subset of AF relay nodes and determine the optimum power allocation per dimension among relays, for a given source power and bandwidth.

Based on this analysis, we compare the energy-efficiency of $\mathrm{DF}$ and $\mathrm{AF}$ in a one-relay network while allowing each strategy to transmit in its optimum bandwidth. We identify the regions where each strategy performs better.

\section{SySTEM MOdEL}

A Gaussian parallel-relay channel is shown in Figure 1. The channel consists of a single source-destination pair and 


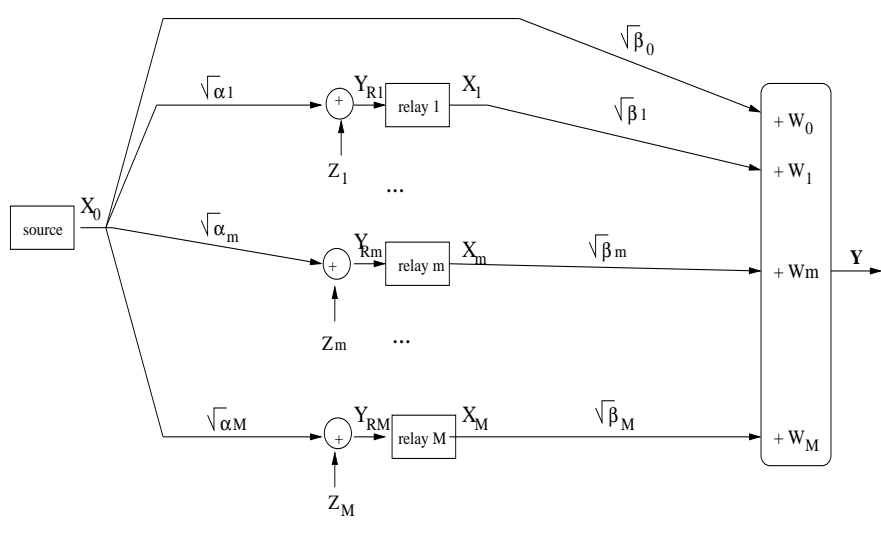

Fig. 1. Parallel-Relay Channel.

$M$ relays that dedicate their resources to relaying information for the source. Each transmission occurs during a transmission interval $T$ in an orthogonal AWGN channel of bandwidth $W$ as in [11]. We view each orthogonal channel as a discrete-time Gaussian channel by representing a waveform of duration $T$ as a vector in the $n=2 W T$ space for sufficiently large $n$ [12]. In each time slot, relay $m$ observes a noisy version of the input $X_{0}$ transmitted at the source

$$
Y_{R m}=\sqrt{\alpha_{m}} X_{0}+Z_{m}
$$

where $Z_{m}$ is a Gaussian random variable with mean zero and variance $N_{0} / 2$ and $\sqrt{\alpha_{m}}$ is the channel gain between the source and relay $m$. Relay $m$ transmits signal $X_{m}$. The destination receives noisy copies of all inputs, as shown in Figure 1. The received signal at the destination at each time slot in a discrete-time channel is

$$
\mathbf{Y}=\mathbf{B X}+\mathbf{W}
$$

where $\mathbf{B}$ is a diagonal channel gain matrix with entries $b_{m m}=$ $\sqrt{\beta_{m-1}}, m=1, \ldots M+1$. Vector $\mathbf{X}=\left[X_{0}, \ldots X_{M}\right]$ is the vector of channel inputs and $\mathbf{W}$ is the Gaussian noise vector with independent components with variance $N_{0} / 2$. For convenience, we assume the units of power are such that $N_{0}=$ 1.

We consider two different transmission strategies at the relays:

- Decode-and-Forward: The transmission from the source is reliably received at a relay. The relay decodes the message, re-encodes it and transmits that message in an orthogonal channel.

- Amplify-and-Forward: The received signal at relay $m$, $1 \leq m \leq M$ is amplified and forwarded in an orthogonal channel. For amplification gain $b_{m} \geq 0$, relay transmits

$$
X_{m}=\sqrt{b_{m}} Y_{R m}=\sqrt{b_{m}}\left(\sqrt{\alpha_{m}} X_{0}+Z_{m}\right) .
$$

\section{OPTIMUM BANDWIDTH}

To formulate the problem, we first consider the achievable rates per dimension for a given power allocation $\mathbf{p}=$ $\left[p_{0}, p_{1} \ldots p_{M}\right]^{T}$ where $p_{0}$ is the source power and $p_{m}$ is the power at relay $m$.
For the given power $p_{0}$ and the rate $r$ at the source, relay $i$ will be able to execute the DF strategy only if the rate $r$ can be communicated reliably from the source to relay $i$ with power $p_{0}$. Thus it has to hold that

$$
W \log \left(1+\frac{\alpha_{i} p_{0}}{W}\right) \geq r .
$$

When constraint (4) is met for node $i$, we say that the source makes node $i$ reliable. Let $A\left(p_{0}\right)$ denote the set of reliable relays, i.e., those relays that are made reliable by a source transmission at power $p_{0}$ and rate $r$, and can thus execute the DF strategy. For given $A\left(p_{0}\right)$, the parallel-relay network (2) is a Gaussian vector channel analogous to a multiple antenna system [13]. The achievable rate per dimension is determined by the maximum mutual information between the channel input $\mathbf{X}$ and output $\mathbf{Y}$. For the DF strategy, it is given by

$$
I_{D F}\left(\frac{\mathbf{p}}{W}\right)=\frac{1}{2} \log \left(1+\frac{p_{0} \beta_{0}}{W}\right)+\sum_{i \in A\left(p_{0}\right)} \frac{1}{2} \log \left(1+\frac{p_{i} \beta_{i}}{W}\right) .
$$

On the other hand, any relay $m$ that is assigned power $p_{m}$ can amplify-and-forward the received signal. The amplification gain $b_{m}$ is chosen such that the transmit power at relay $m$ is $p_{m}$ and is found from (3) to be

$$
b_{m}=\frac{p_{m}}{\alpha_{m} p_{0}+W} \quad m=1, \ldots M .
$$

We can again use the result for Gaussian vector channels to determine the achievable rate for the AF strategy

$I_{A F}\left(\frac{\mathbf{p}}{W}\right)=\frac{1}{2} \log \left[1+\frac{p_{0}}{W}\left(\beta_{0}+\sum_{m=1}^{M} \frac{\alpha_{m} \beta_{m} p_{m}}{\alpha_{m} p_{0}+\beta_{m} p_{m}+W}\right)\right]$.

The following observation motivates the formulation of our problem.

Lemma 1: For any given power allocation $\mathbf{p}, W I_{D F}(\mathbf{p} / W)$ is increasing in $W$ for the decode-and forward strategy. For the amplify-and-forward strategy, there exists finite $W^{*}$ that maximizes $W I_{A F}(\mathbf{p} / W)$.

Lemma 1 states the well known fact that the energy cost per information bit for the DF strategy is maximized when the network operates in the wideband regime [14]. On the other hand, Lemma 1 shows that the optimum operating bandwidth $W^{*}$ for the AF strategy is finite. Consider the maximum achievable rate in bits/s at the destination node for the $\mathrm{AF}$ strategy

$$
C=2 W I_{A F}\left(\frac{\mathbf{p}}{W}\right) \quad \text { bits/s }
$$

where $I_{A F}$ is given by (7). For large $W$,

$$
\lim _{W \rightarrow \infty} C=\frac{\beta_{0} p_{0}}{\ln 2}
$$

which is the rate achieved in the wideband regime by direct transmission from the source. Therefore, when operating in the wideband regime, there is no benefit from transmissions at relays that employ AF strategy. This behavior was previously observed in [2] in a parallel Gaussian network with two relays. With this observation in mind, we consider the problem to maximize the achievable rate in the network under the given power constraint. Rather than considering the power 
constraint imposed on each transmitter, we consider a less restrictive power constraint on the total power in the network $E\left[\mathbf{X}^{\mathbf{T}} \mathbf{X}\right] \leq p / 2 W$. Because $\mathrm{DF}$ and $\mathrm{AF}$ will not in general operate in the same bandwidth, we compare the performance of two strategies, using the total power constraint

$$
\mathbf{1}^{T} \mathbf{p} \leq p .
$$

The problem of maximizing the achievable rate under the total power constraint (10) has its dual with the objective to communicate to the destination at rate $r$ bits/s using minimum transmitted power $p^{*}$. The two problems have analogous solutions that become the same when $p^{*}=p$. In the rest of the paper we present the solution to the latter problem.

\section{DECODE-AND-FORWARD}

When transmitting with rate $r$, the source will make node $i$ reliable if constraint (4) is met. The achieved rate at the destination is then given by (5). This implies that, for the source to communicate at rate $r$ with the destination, we require

$$
W \log \left(1+\frac{p_{0} \beta_{0}}{W}\right)+\sum_{i \in A\left(p_{0}\right)} W \log \left(1+\frac{p_{i} \beta_{i}}{W}\right) \geq r .
$$

To optimize the transmit powers, we have to find the best subset of nodes to be made reliable so that they can decode-andforward the message. We use binary variables $x_{i}$ to indicate which relays will be reliable and formulate our problem in the following way:

$$
\min \sum_{i=0}^{M} p_{i}
$$

subject to

$$
\begin{gathered}
W \log \left(1+\frac{p_{0} \beta_{0}}{W}\right)+\sum_{i=1}^{M} x_{i} W \log \left(1+\frac{p_{i} \beta_{i}}{W}\right) \geq r \\
W \log \left(1+\frac{p_{0} \alpha_{i}}{W}\right)>x_{i} r \\
x_{i} \in\{0,1\}, \\
p_{i} \geq 0
\end{gathered}
$$

In the limit of large $W$, this problem simplifies to the wideband DF relay problem:

$$
\begin{aligned}
\min \sum_{i=0}^{M} p_{i} & \\
\text { subject to } p_{0} \beta_{0}+\sum_{i=1}^{M} x_{i} p_{i} \beta_{i} & \geq r, \\
p_{0} \alpha_{i} & >x_{i} r, \\
x_{i} & \in\{0,1\}, \\
p_{i} & \geq 0 .
\end{aligned}
$$

Theorem 1: The wideband DF relay problem (14) admits an optimal solution in which no more than one relay node transmits.
Theorem 1 holds because when transmitting with severely restricted transmitter power, water filling over the relays results in transmitting to the relay with the best channel to the destination.

By Theorem 1, it is sufficient to consider only policies that employ a single relay $k$. In this case, the problem (14) becomes the wideband single relay problem

$$
\begin{aligned}
& \min p_{0}+p_{k} \\
& \text { subject to } p_{0} \beta_{0}+x_{k} p_{k} \beta_{k} \geq r, \\
& p_{0} \alpha_{k} \geq x_{k} r, \\
& x_{k} \in\{0,1\}, \\
& p_{0}, p_{k} \geq 0 .
\end{aligned}
$$

In the problem (15), one can show that relay $k$ is used only if $\alpha_{k}>\beta_{0}$ and $\beta_{k}>\beta_{0}$. In this case, the transmit powers are

$$
p_{0}^{*}=\frac{r}{\alpha_{k}}, \quad p_{k}^{*}=\frac{r-\beta_{0} r / \alpha_{k}}{\beta_{k}} .
$$

The total transmitted power of this solution is

$$
p_{0}^{*}+p_{k}^{*}=r\left[\frac{1}{\alpha_{k}}+\frac{1}{\beta_{k}}-\frac{\beta_{0}}{\alpha_{k} \beta_{k}}\right] \text {. }
$$

We emphasize that this is the optimal power assignment for using node $k$ as long as node $k$ is a useful relay, in the sense that $k$ belong to the set of useful relays

$$
U=\left\{i \mid \alpha_{i}>\beta_{0}, \beta_{i}>\beta_{0}\right\} .
$$

Finally, among all useful relays $k$, we choose that one which minimizes the total transmitted power $p_{0}^{*}+p_{k}^{*}$. We summarize our observations in the following theorem.

Theorem 2: If the set $U$ of useful relays is non-empty, the optimal solution to the wideband DF relay problem (14) is for the source to employ relay

$$
k^{*}=\arg \min _{k \in U}\left[\frac{1}{\alpha_{k}}+\frac{1}{\beta_{k}}-\frac{\beta_{0}}{\alpha_{k} \beta_{k}}\right]
$$

with power assignment given by (16); otherwise, if $U$ is empty, then direct transmission from the source to the destination is optimal.

\section{AMPLIFY-AND-FORWARD}

We now define the power minimization problem for the AF strategy. We formulate the problem in terms of the power per dimension $\mathbf{P}=\mathbf{p} / W$. The achievable rate (7) becomes

$I_{A F}(\mathbf{P})=\frac{1}{2} \log \left[1+P_{0}\left(\beta_{0}+\sum_{m=1}^{M} \frac{\alpha_{m} \beta_{m} P_{m}}{\alpha_{m} P_{0}+\beta_{m} P_{m}+1}\right)\right]$.

Since the optimum bandwidth in this case is not known, we formulate the AF relay problem as

$$
\begin{aligned}
& p^{*}=\min _{\mathbf{P}, W} 2 W \mathbf{1}^{T} \mathbf{P} \\
& \text { subject to } 2 W I_{A F}(\mathbf{P}) \geq r \\
& \mathbf{P} \geq 0 \\
& 0 \leq W \leq W_{\max } .
\end{aligned}
$$


We assume that $W_{\max }$ is a maximum bandwidth available in each channel. We choose $W_{\max }$ sufficiently large to allow the network to operate in the wideband regime. Let $\left(\mathbf{P}^{*}, W^{*}\right)$ denote the optimum power and bandwidth allocation that achieves $p^{*}$ in (21). We first observe that, to achieve nonzero rate in (21) and thus satisfy the rate constraint (21a), the network uses bandwidth $W^{*}>0$ and the source transmits with power $P_{0}^{*}>0$. Furthermore, constraint (21a) is always binding. Depending on the values of the channel gains, a solution to problem (21) may be a direct transmission from the source, that is, $P_{m}^{*}=0$ for all $m=1, \ldots M, W^{*}=W_{\max }$ and $P_{0}^{*}$ given by (21a). Otherwise, there is a subset of $K \leq M$ relays employing the AF strategy.

The Lagrangian in Problem (21) is

$$
\Lambda=2 W \sum_{m=0}^{M} P_{m}-\lambda\left(2 W I_{A F}(\mathbf{P})-r\right)
$$

We next characterize the optimum bandwidth for the AF strategy in (21).

Lemma 2: For $W_{\max }$ sufficiently large, the optimum bandwidth for the AF strategy in (21) is strictly smaller than $W_{\max }$. Proof for Lemma 2 follows from the fact that, for any fixed $\mathbf{p}, W I_{A \boldsymbol{F}}(\mathbf{p} / W)$ is decreasing in $W$ for large $W$.

Given $\mathbf{P}^{*}$ for the AF strategy, it would be convenient to relabel the nodes such that $1, \ldots, K$ relays are the active transmitters with powers $P_{m}^{*}>0$ and $P_{m}^{*}=0, \quad m=K+$ $1, \ldots, M$. From Lemma 2, the optimum solution for the AF strategy in (21) is never on the boundary of constraint (21c), that is $0<W^{*}<W_{\max }$. By the Kuhn-Tucker conditions, this implies

$$
\frac{\partial \Lambda}{\partial W}=2 \sum_{m=0}^{K} P_{m}^{*}-2 \lambda I_{A F}\left(\mathbf{P}^{*}\right)=0
$$

From (23), we obtain the Lagrange multiplier

$$
\lambda=\frac{\sum_{m=0}^{K} P_{m}^{*}}{I_{A F}\left(\mathbf{P}^{*}\right)} .
$$

For nodes $k=0, \ldots K$ with non-zero transmitter powers, the Kuhn-Tucker conditions are

$$
\frac{\partial \Lambda}{\partial P_{k}}=2 W^{*}\left[1-\lambda \frac{\partial I_{A F}\left(\mathbf{P}^{*}\right)}{\partial P_{k}}\right]=0 \quad k=0, \ldots, K .
$$

From (24) and (25),

$$
\frac{\partial I_{A F}\left(\mathbf{P}^{*}\right)}{\partial P_{k}}=\frac{1}{\lambda}=\frac{I_{A F}\left(\mathbf{P}^{*}\right)}{\sum_{m=0}^{K} P_{m}^{*}} \quad k=0, \ldots K .
$$

The optimum power allocation $\left(P_{0}^{*}, \ldots, P_{K}^{*}\right)$ can then be determined from $K+1$ equations given by (26), independent of $r$ and $W^{*}$. The optimum bandwidth can be determined such that the solution lies on the feasibility region boundary (21a):

$$
W^{*}=\frac{r}{2 I_{A F}\left(\mathbf{P}^{*}\right)}
$$

where the first $K+1$ elements of $\mathbf{P}^{*}$ are nonzero and are given by $\left(P_{0}^{*}, P_{1}^{*}, \ldots, P_{K}^{*}\right)$ and the rest $M-K+1$ elements are zero. From (21), (24) and (27),

$$
p^{*}=2 W^{*} \sum_{m=0}^{K} P_{m}^{*}=\frac{\sum_{m=0}^{K} P_{m}^{*}}{I_{A F}\left(\mathbf{P}^{*}\right)} r=\lambda r .
$$

We thus proved the following:

Theorem 3: The AF relay problem (21) has an optimum solution in which the optimum bandwidth $W^{*}$, rate $r$ and the total transmit power $p^{*}$ have a linear relationship.

We can view $\lambda$ as a 'power price': increasing the required rate $r$ in (21) by $\Delta r$, increases the minimum required total power $p^{*}$ by $\lambda \Delta r$.

The optimum solution to the AF relay problem (21) is specified by the optimum transmit powers at the source and the relays, operating in the optimum bandwidth. To determine the optimum power allocation requires solving the system of equations given by (26) which does not appear as an easy task. We approach this problem by considering a subproblem of (21) that determines the optimum power allocation per dimension among relays only, for a given source power and bandwidth.

\section{A. Optimum Power Allocation at Relays}

To identify the best subset of AF relay nodes for any source power and signaling bandwidth, we let,

$$
\gamma_{m}=\sqrt{\frac{\alpha_{m} \beta_{m}}{\alpha_{m} P_{0}+1}}
$$

The achievable rate per dimension (20) becomes

$$
I_{A F}(\mathbf{P})=\frac{1}{2} \log \left[1+P_{0}\left(\beta_{0}+\sum_{m=1}^{M} \frac{\alpha_{m} \gamma_{m}^{2} P_{m}}{\alpha_{m}+\gamma_{m}^{2} P_{m}}\right)\right] .
$$

For fixed bandwidth and power at the source, maximizing $I_{A F}(\mathbf{P})$ over the choice of relay powers is equivalent to

$$
\begin{array}{r}
\max _{\hat{\mathbf{P}}} \sum_{m=1}^{M} \frac{\alpha_{m} \gamma_{m}^{2} P_{m}}{\alpha_{m}+\gamma_{m}^{2} P_{m}} \\
\text { subject to } \sum_{m=1}^{M} P_{m} \leq P_{R}, \\
\hat{\mathbf{P}} \geq 0 .
\end{array}
$$

where $\hat{\mathbf{P}}=\left[P_{1}, \ldots, P_{M}\right]$ is the vector of relay transmit powers. The power per dimension allocated to relays is

$$
P_{R}=\frac{p}{W}-P_{0}
$$

where $p$ is the total power constraint as given by (10). Note that (31) solves the dual to (21) and for $p=p^{*}$ the two problems have identical solutions. From the Kuhn-Tucker conditions, the optimum solution to (31) is

$$
P_{m}^{*}=\frac{\alpha_{m}}{\gamma_{m}}\left[\frac{1}{\sqrt{\eta}}-\frac{1}{\gamma_{m}}\right]^{+} \quad m=1, \ldots K
$$

where $\eta$ is the Lagrange multiplier and can be found such that boundary constraint (31a) is satisfied with equality. When solving (31) for optimum $\left(W^{*}, P_{0}^{*}\right)$, from (32) and (31a) it follows that $\sum_{m=1}^{M} \hat{P}_{m}^{*}=p^{*} / W^{*}-P_{0}^{*}$, and thus $\hat{\mathbf{P}}^{*}$ solves (21). Alternatively, we could derive solution (33) directly from (25).

From (33) we observe that the optimum AF strategy in general employs a set of relays, unlike the optimum DF strategy. The optimum choice of relays strongly depends on 


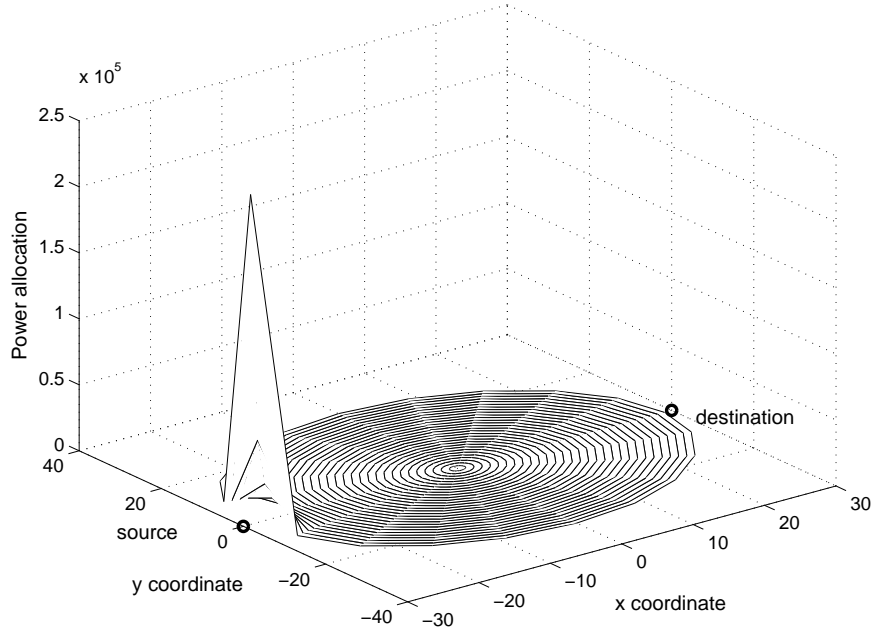

Fig. 2. Power allocation among relays when operating in low SNR at the source

the source transmit power. Figures 2 and 3 show the optimum power allocation among the relays spread over an area in between source and destination, for two significantly different source powers. For a source operating at low-SNR, Figure 2 shows that the best choice is to relay through the nodes that are close to the source. This is not surprising since, in this case, the "broadcast" part of the channel from the source to relays is weak. The solution thus tries to maximize the performance of the broadcast side by choosing nodes that have the highest received SNR. The situation is opposite in the high SNR regime at the source, shown in Figure 3, where the SNR at the relays is high and relay-destination channel limits the performance of the network. The solution then choses relays that have better channel to the destination.

Thus, the best choice of relay nodes significantly depends on the transmit power at the source and our next goal is to solve (21) and determine the optimum operating point.

For two limiting power regimes, it follows from (29) that the parameter $\gamma_{m}$ is determined by the channel gains as

$$
\gamma_{m}= \begin{cases}\sqrt{\alpha_{m} \beta_{m}}, & \text { in low-SNR regime } \\ \sqrt{\beta_{m} / P_{0}}, & \text { in high-SNR regime }\end{cases}
$$

and is thus independent from $P_{0}$ when source is operating in low-SNR regime. On the other hand, from (33), a node $m$ will be select as a relay when $\gamma_{m}>\sqrt{\eta}$, for any given choice of bandwidth and source power $P_{0}$. Increasing $P_{0}$ will have an effect of increasing $\eta$ and thus of shutting down relays in the increasing order of $\gamma_{m}$. The similar effect will occur when source is operating in high-SNR regime. This observation can then be used to perform the search for the optimum power. Finally, we compare the energy-efficiency of DF and AF supporting the fixed rate $r$ in the one-relay network. For DF, the network operates in the wideband regime. The total power for $\mathrm{DF}$ is given by (17). For AF, for a given bandwidth $W$, the optimum power allocation per dimension is determined from (21) and the optimum bandwidth is found numerically. The comparison of the power-efficiency is shown in Figure 4.

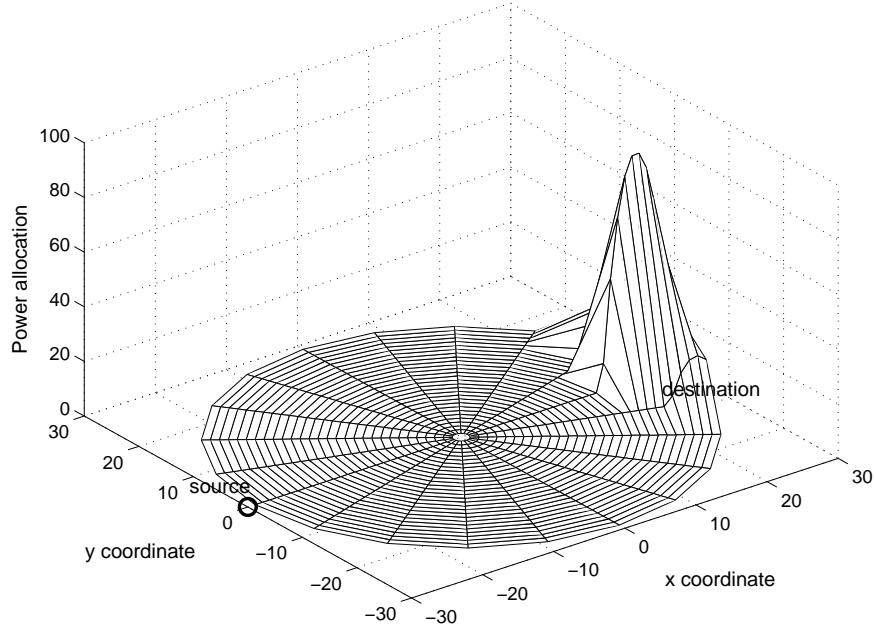

Fig. 3. Power allocation among relays when operating in high SNR at the source

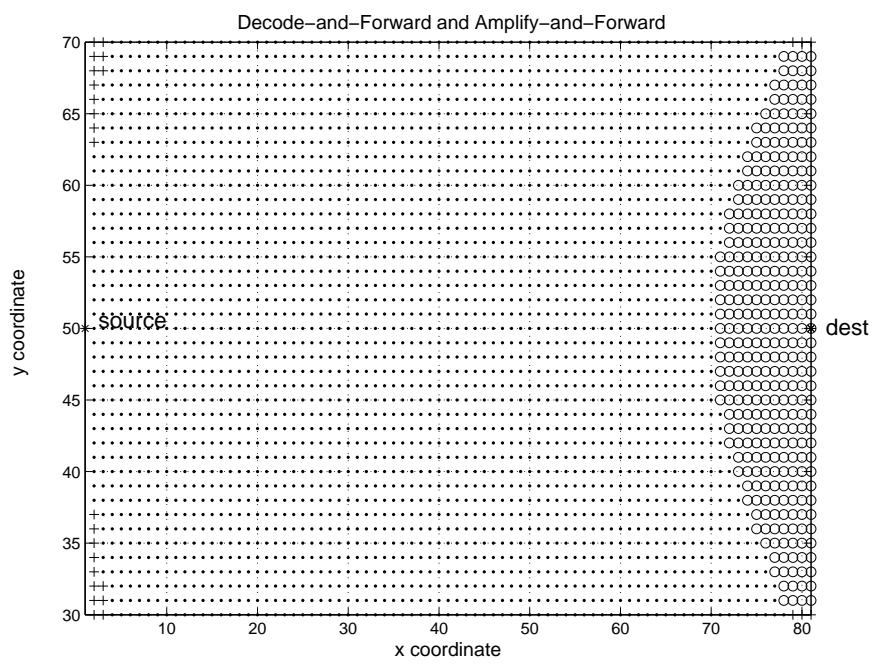

Fig. 4. Decode-and-forward and amplify-and-forward performance.

The dots, circles and pluses indicate, respectively, the regions where $\mathrm{DF}, \mathrm{AF}$ and direct transmission support the rate $r$ with minimum power.

\section{REFERENCES}

[1] S. Verdú, "On channel capacity per unit cost," IEEE Trans. on Information Theory, vol. 36, no. 5, pp. 1019-1030, Sept. 1990.

[2] B. E. Schein, "Distributed coordination in network information theory," in Ph.D thesis, Massachusetts Institute of Technology, Sept. 2001.

[3] T. Cover and A. E. Gamal, "Capacity theorems for the relay channel," IEEE Trans. on Information Theory, vol. 25, no. 5, pp. 572-584, Sept. 1979.

[4] M. Gastpar, "To code or not to code," in Ph.D. Thesis, Swiss Federal Institute of Technology (EPFL), Lausanne, Switzerland, Nov. 2002.

[5] M. Gastpar and M. Vetterli, "On asymptotic capacity of gaussian relay networks," in Proc. of International Symposium on Information Theory (ISIT'02), June 2002.

[6] M. Gastpar, G. Kramer, and P. Gupta, "The multiple-relay channel: Coding and antenna-clustering capacity," in Proc. of International Symposium on Information Theory (ISIT'02), June 2002.

[7] P. Gupta and P. R. Kumar, "Towards an information theory of large networks: An achievable rate region," IEEE Trans. on Information Theory, vol. 49, pp. 1877-1894, Aug. 2003.

[8] G. Kramer, M. Gastpar, and P. Gupta, "Capacity theorems for wireless 
relay channels," in Proc. of the Allerton Conference on Communications, Control and Computing, Monticello, IL, Oct. 2003.

[9] G. Caire, D. Tuninetti, and S. Verdú, "Suboptimality of TDMA in the low-power regime," IEEE Trans. on Information Theory, submitted, Sept. 2002.

[10] D. Tse and S. Hanly, "Multi-access fading channels: Part i: Polymatroid structure, optimal resource allocation and throughput capacities," IEEE Trans. on Information Theory, vol. 45, no. 2, pp. 2796-2815, Nov. 1998.

[11] J. N. Laneman, D. N. C. Tse, and G. W. Wornell, "Cooperative diversity in wireless networks: efficient protocols and outage behavior," IEEE Trans. on Information Theory, submitted.

[12] T. Cover and J. Thomas, Elements of Information Theory. John Wiley Sons, Inc., 1991.

[13] E. Telatar, "Capacity of multi-antenna gaussian channels," in Europ. Trans. Telecommunications, Nov. 1997.

[14] S. Verdú, "Spectral efficiency in the wideband regime," IEEE Trans. on Information Theory, vol. 48, no. 6, pp. 1319-1343, June 2002. 